\title{
Influencia de la variación de la presión de oxígeno ambiental en la formación ósea en cuyes nativos del nivel del mar
}

\author{
Influence of environmental oxygen pressure variation in sea level native guinea pigs bone
}

formation

\author{
Elías Ernesto Aguirre Siancas ${ }^{1,2,3, a, b, c}$ \\ 'Facultad de Odontología, Universidad Nacional Mayor de San Marcos, Lima, Perú. \\ ${ }^{2}$ Facultad de Farmacia y Bioquímica, Universidad Nacional Mayor de San Marcos, Lima, Perú. \\ ${ }^{3}$ Facultad de Ciencia de la Salud, Universidad Científica de Sur, Lima, Perú. \\ ${ }^{a}$ Cirujano Dentista; ${ }^{b}$ Magister en Fisiología; ${ }^{\mathrm{C}}$ Miembro de la Sociedad Brasileña de Fisiología.
}

\begin{abstract}
Resumen
Introducción: La hipoxia caracteriza a los tejidos inicialmente injuriados; la variación en la disposición del oxígeno tisular en el proceso de reparación ósea determina la aparición de diversas moléculas implicadas en la sanación del tejido. Objetivos: Determinar si la variación de la presión de oxígeno ambiental influirá en la formación ósea posterior a una osteotomía en cuyes nativos del nivel del mar. Diseño: Experimental. Lugar: Facultad de Odontología de la Universidad Nacional Mayor de San Marcos, Lima, Perú, e Instituto Veterinario de Investigaciones Tropicales y de Altura, Mantaro, Perú. Material biológico: Cuyes. Intervenciones: Se utilizó 5 grupos de 10 cuyes cada uno, uno sin inducción de osteotomía (grupo control) y cuatro grupos experimentales: mar 15 días y mar 30 dias (expuestos a PO2 ambiental de $157 \mathrm{mmHg}$ ), altura 15 dias y altura 30 dias (expuestos a PO2 ambiental de $107 \mathrm{mmHg}$ ). Principales medidas de resultados: Conteo de osteocitos. Resultados: El grupo mar 15 dias presentó menor número de osteocitos comparado con el grupo altura 15 días (63 180 vs. 80 310, p<0,05). A su vez, el grupo mar 30 días presentó también menor número de osteocitos comparado con el grupo altura 30 dias (160 640 vs. 167 370, p<0,05). Conclusión: La menor presión de oxígeno ambiental favoreció una mayor formación ósea en cuyes nativos del nivel mar.
\end{abstract}

Palabras clave: Presión de oxígeno ambiental, formación ósea, osteocitos.

\section{Abstract}

Introduction: Hypoxia characterizes initially injured tissue; variation in oxygen available determines the appearance of molecules involved in bone repair. Objectives: To determine if environmental oxygen pressure variation influences bone formation following osteotomy in sea level native guinea pigs. Design: Experimental. Setting: Faculty of Dentistry, Universidad Nacional Mayor de San Marcos, Lima, Peru, and Tropical and High Altitude Research Veterinary Institute, Mantaro, Peru. Biological material: Guinea pigs. Interventions: Five groups of 10 guinea pigs each included one without osteotomy (control group) and four experimental groups: sea 15 days and sea 30 days (exposed to environmental PO2 $157 \mathrm{mmHg}$ ), high altitude 15 days and high altitude 30 days (exposed to environmental PO2 $107 \mathrm{mmHg}$ ). Main outcome measures: Osteocytes count. Results: The 15 days sea group had lower number of osteocytes compared with the 15 days high altitude group (63 180 vs. $80310, p<0.05$ ). The 30 days sea group had also lower number of osteocytes compared with the 30 days high altitude group (160 640 vs. 167 370, p<0.05). Conclusions: The lower environmental oxygen pressure favored higher bone formation in sea level native guinea pigs.

Keywords: Ambiental oxygen pressure, bone formation, osteocytes.

An Fac med. 2014;75(2):125-9 / doi: http://dx.doi.org/10.15381/anales.v75i2.8351

\section{INTRODUCCIÓN}

El tejido óseo está constituido por un conjunto de células y por una matriz extracelular calcificada. En condiciones basales, dichos componentes se encuentran en constante mecanismo de remodelación: formación y resorción ${ }^{(1)}$. Cuando ocurre una lesión ósea, como una fractura o una osteotomía, los mecanismos de formación predominan, dado que el tejido lesionado requiere una recuperación eficaz. En este proceso de reparación ósea, las células osteoprogenitoras y los osteoblastos cumplen un papel cardinal (2). La hipoxia es una condición inicial de este microambiente injuriado; la disminución de oxígeno induce la proliferación de múltiples tipos celulares y determina procesos complejos en los cuales están involucradas un gran número de moléculas, entre las que destacan: el factor inducible por hipoxia (HIF), el factor de crecimiento vascular del endotelio (VEGF), el RUNX2, el osterix (Osx), el factor de crecimiento de fibroblastos (FGF), entre otras ${ }^{(3-8)}$.
El factor inducible por hipoxia (HIF) es un factor de transcripción constituido por la subunidad HIF- $\alpha$, de ubicación citosólica, y la subunidad HIF-1 $\beta$, de ubicación nuclear; presentan pesos moleculares de $120-130 \mathrm{KDa}$ y $91-94$ $\mathrm{KDa}$, respectivamente. En normoxia tisular, la HIF- $\alpha$ es hidroxilada por las enzimas propil (tipo PHD-2) y la asparagil (tipo FIT-1) hidroxilasas y luego es marcada por una ubiquitina, la proteína de von Hippel-Lindau (pVHL); finalmente es degradada por el proteosoma. Las enzimas hidroxilasas requieren 
oxígeno para sus funciones; por ello, en normoxia la HIF- $\alpha$ es continuamente degradada. Pero, cuando la disposición tisular disminuye (hipoxia), las enzimas se inactivan y la subunidad HIF- $\alpha$ empieza a acumularse rápidamente en el citosol ${ }^{(7,9,10)}$. Por otra parte, la HIF-1 $\beta$ es constitutiva e independiente de la disposición de oxígeno tisular. Cuando en promedio la concentración de oxígeno tisular disminuye por debajo de $5 \%$, la HIF- $\alpha$ se incrementa y se desplaza al núcleo, acoplándose con la HIF-1 $\beta$ y empezando así la transcripción de sus genes objetivos ${ }^{(7,11,12)}$. Dentro de estos genes destacan los que codifican la eritropoyetina, la transferrina, el transportador de glucosa 1 y 3 , y el factor de crecimiento vascular del endotelio (VEGF), el cual es indispensable en la vascularización y en la diferenciación osteoblástica ${ }^{(9,13)}$.

La $\mathrm{PO}_{2}$ varía fisiológicamente entre los distintos tipos celulares. El aire ambiental contiene una concentración

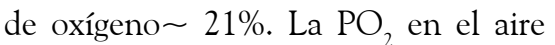
inspirado progresivamente disminuye después de entrar por los pulmones. Por esta razón, en la mayoría de los tejidos internos los niveles de $\mathrm{PO}_{2}$ varían entre 14 y $65 \mathrm{mmHg}\left(2 \%\right.$ a $9 \%$ de $\left.\mathrm{O}_{2}\right)$. Siendo el rango normal de la $\mathrm{PO}_{2}$ en la médula ósea entre 15,2 y 45,6 $\mathrm{mmHg}$, en las zonas de reparación de hueso se puede encontrar entre 5,3 y $78,9 \mathrm{mmHg}(0,7$ a $10,3 \%$ de $\left.\mathrm{O}_{2}\right)^{(4,14)}$. La tensión de oxígeno en el aire ambiental a nivel del mar es de $157 \mathrm{mmHg}\left(\sim 21 \%\right.$ de $\left.\mathrm{O}_{2}\right)$ y se considera típicamente como normoxia; sin embargo, la concentración de oxígeno entre $2 \%$ y $9 \%$ ha sido recientemente apreciada por algunos científicos como normoxia fisiológica $(4,14,15)$.

En la actualidad, son múltiples los estudios que evalúan las bases moleculares en los procesos metabólicos del tejido óseo, tanto en normoxia como en hipoxia, encontrándose diferentes resultados. Algunos concluyen que la diferenciación osteoblástica, la expresión de genes pro-osteogénicos y la resultante formación ósea están más aumentadas en tejidos expuestos a ambientes bajo normoxia, $\sim 21 \%$ de $\mathrm{O}_{2}{ }^{(15-18)}$; por otra parte, en otros estudios, dichos indicadores están más aumentados en tejidos expuestos a ambientes bajo hipoxia, $\sim 1-5 \%$ de $\mathrm{O}_{2}{ }^{(4,19-21)}$. En base a estos resultados y a la necesidad de aportar a la clarificación de bajo qué concentración de $\mathrm{O}_{2}$ el tejido óseo tiene una mejor formación, el presente trabajo tuvo como objetivo evaluar la influencia de la presión de oxígeno ambiental sobre la formación ósea posterior a una osteotomía del maxilar inferior, en cuyes nativos del nivel del mar.

\section{MÉTODOS}

Se trabajó con 50 cuyes machos de la raza Perú, de 400 a 450 gramos de peso corporal y de 5 a 8 semanas de edad, obtenidos en la Facultad de Medicina Veterinaria de la Universidad Nacional Mayor de San Marcos (UNMSM). El tamaño muestral fue tomado en base a estudios similares ${ }^{(22-24)}$. Los cuyes recibieron alimento y agua ad líbitum, con ciclos luz/oscuridad de 12 horas alternadas, en el Laboratorio de Fisiología de la Facultad de Odontología de la UNMSM y en el Instituto Veterinario de Investigaciones Tropicales y de Altura - Mantaro (IVITA-Mantaro). Se siguió la guía de ética para animales de experimentación Guide for the Care and Use of Laboratory Animals ${ }^{(25)}$.

Se asignó de forma aleatoria 5 grupos conformados por 10 cuyes cada uno: 1) grupo control, sacrificado el día 1 , sin procedimiento quirúrgico; 2) grupo mar 15 días, sometido a la $\mathrm{PO}_{2}$ de $157 \mathrm{mmHg}$ y sacrificado a los 15 días del procedimiento quirúrgico; 3) grupo mar 30 días, sometido a la $\mathrm{PO}_{2}$ de $157 \mathrm{mmHg}$ y sacrificado a los 30 días del procedimiento quirúrgico; 4) grupo altura 15 días, sometido a la $\mathrm{PO}_{2}$ de 107 mmHg y sacrificado a los 15 días del procedimiento quirúrgico; y, 5) grupo altura 30 días, sometido a la $\mathrm{PO}_{2}$ de 107 mmHg y sacrificado a los 30 días del procedimiento quirúrgico.

El día 1 en cada cuy de los grupos mar 15 días, mar 30 días, altura 15 días y altura 30 días se realizó el procedi- miento quirúrgico de osteotomía que consistió, primeramente, en la inducción con anestesia general usando ketamina (20 mg/kg de peso vía IM) ${ }^{(26)}$. Luego, en el borde mandibular derecho del animal se infiltró localmente lidocaína al 2\% para reforzar la analgesia. Con una hoja de bisturí $\mathrm{N}^{\circ} 15$ se hizo una incisión lineal de $1,5 \mathrm{~cm}$, se decoló y se expuso el hueso mandibular, completándose el procedimiento con el desgaste óseo, para lo que se empleó una piedra dental redonda de carburo tungsteno marca Maileffer, fresándose por 5 segundos. Finalmente, con 2 puntos simples de catgut crómico 4 ceros se suturó la herida.

Una vez sacrificados los animales (el día 1 en el grupo control, y a los 15 o 30 días en los grupos experimentales), se obtuvo las muestras de la zona mandibular tratada por biopsia quirúrgica. Dichas muestras fueron fijadas en formol al 10\% y enviadas al laboratorio, en el que se empleó coloración de hematoxilina-eosina. Para la medición del indicador (conteo de osteocitos), se procedió a identificar, específicamente, la zona intervenida mediante microscopia; luego, se observó tres campos de dicha zona tomados al azar; esto se realizó en cada una de las láminas obtenidas de cada cuy. De las 3 observaciones se obtuvo la media, la cual fue el número de osteocitos para dicho cuy ${ }^{(8,22,27)}$. Cada área microscópica observada fue de $0,458 \mathrm{~mm}^{2}$, con aumento total de 400X, empleándose un equipo marca Micros modelo MCX100 LCD Crocus II.

Para el análisis estadístico se utilizó el paquete SPSS versión 19 para Windows. Los datos son presentados en tablas y figuras como media $+/$ - desviación estándar. Las pruebas se realizaron con un nivel de significancia de $95 \%$. Mediante la prueba de normalidad de Kolmogorov-Smirnov se determinó que los datos seguían una distribución normal. El contraste de hipótesis fue mediante la prueba $t$ de Student, el análisis de varianza Anova y comparaciones múltiples con la prueba de Tukey. 


\section{RESULTADOS}

Los hallazgos del estudio histopatológico (indicador: conteo de osteocitos) mostraron que la variación de la presión de oxígeno ambiental produjo una mayor formación ósea en el grupo altura 15 días comparado con el grupo mar 15 días (tabla 1), lo cual fue estadísticamente significativo. A su vez, al comparar el grupo mar 30 días versus el grupo altura 30 , hubo tendencia a mayor formación ósea en el grupo expuesto a $107 \mathrm{mmHg}$, pero dichos resultados no fueron estadísticamente significativos (tabla 2).

Al evaluar el proceso de formación ósea en cada una de las presiones de oxígeno estudiadas (157 mmHg y107 $\mathrm{mmHg}$ ), se encontró que la cantidad de osteocitos fue mayor en el grupo mar 30 días (160 $640 \pm 12990)$ y en el grupo altura 30 días (167 $370 \pm 13$ 059) al compararlos con el grupo mar 15 días (63 $180 \pm 11323$, figura 1$)$ y con el grupo altura 15 (80 $310 \pm 9336$ osteocitos, figura 2), respectivamente; siendo los resultados estadísticamente significativos.

\section{DISCUSIÓN}

Mientras el rol del oxígeno sobre los mecanismos celulares en la formación ósea ha sido estudiado extensamente in vitro, poco es el conocimiento acerca de la relevancia de dichos estudios in vivo. La mayoría de estudios usan como condición hipóxica concentraciones de oxígeno de 1 a $5 \%$, que resultan ser comparados con cultivos con concentraciones de oxígeno de $18 \%$ a $21 \%$, considerados como condición normóxica. In vivo, los múltiples tipos celulares con sus diferentes demandas metabólicas hacen mucho más complicados los trabajos de investigación. La interacción de la variedad de factores de crecimiento y citoquinas en los procesos de reparación, y la complejidad de estos determinantes in vivo, aparte de las variables intervinientes ambientales, impiden una evaluación completa de todo el proceso en los diversos trabajos ${ }^{(23)}$.
Tabla 1. Grupos sometidos a diferentes presiones de oxígeno ambiental evaluados a los 15 días post-operación.

\begin{tabular}{llcccc} 
& $\begin{array}{l}\text { Grupos evaluados según presión } \\
\text { aplicada }\end{array}$ & N & Media & $\begin{array}{c}\text { Desviación } \\
\text { típica }\end{array}$ & $p$ \\
\hline Grupo de osteocitos & Grupo mar 15 días $(157 \mathrm{mmHg})^{\text {a }}$ & 10 & 63180 & 11323 & 0,002 \\
& Grupo altura 15 días $(107 \mathrm{mmHg})^{\text {b }}$ & 10 & 80310 & 9336 & \\
\hline
\end{tabular}

${ }^{a, b}$ Superindices diferentes significan resultados estadisticamente significativos.

Tabla 2. Grupos sometidos a diferentes presiones de oxígeno ambiental evaluados a los 30 días post-operación.

\begin{tabular}{llcccc} 
& $\begin{array}{l}\text { Grupos evaluados según presión } \\
\text { aplicada }\end{array}$ & N & Media & $\begin{array}{c}\text { Desviación } \\
\text { típica }\end{array}$ & $p$ \\
\hline Grupo de osteocitos & Grupo mar 30 días $(157 \mathrm{mmHg})$ & 10 & 160640 & 12990 & 0,263 \\
& Grupo altura 30 días $(107 \mathrm{mmHg})$ & 10 & 167370 & 13059 & \\
\hline
\end{tabular}

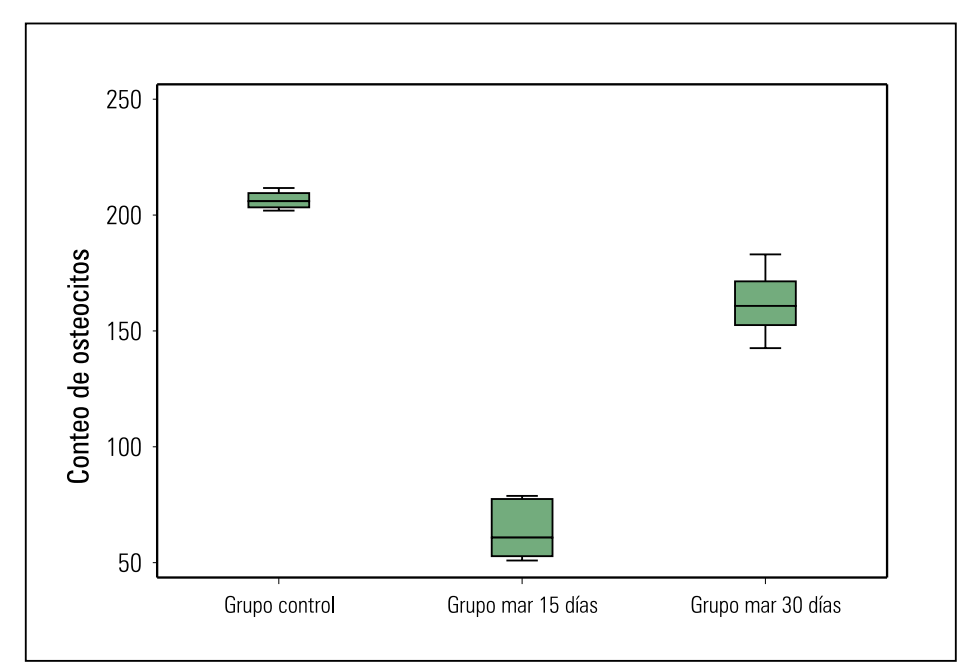

Figura 1. Evaluación del proceso de formación ósea a la presión de oxígeno ambiental de $157 \mathrm{mmHg}$ (150 msnm).

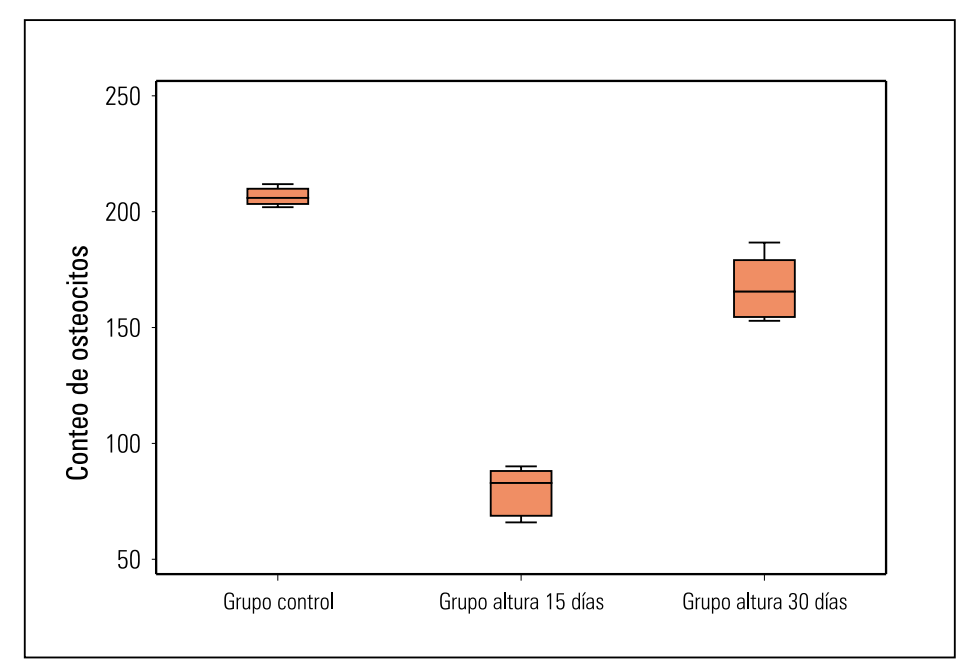

Figura 2. Evaluación del proceso de formación ósea a la presión de oxígeno ambiental de 107 mmHg (3320 msnm). 
A pesar de ser la altitud una condición geográfica que determina variaciones en la temperatura, en la humedad, en la radiación solar y en la presión de oxígeno ambiental, en el presente trabajo se ha evaluado la influencia de la presión de oxígeno ambiental sobre la formación ósea por ser esta variable la más importante y la más estudiada, por las profundas modificaciones que origina en la fisiología de los seres vivos. La reducción en la disposición de oxígeno ambiental produce una menor inspiración de este por los pulmones; la hipoxia induce la actividad del factor inducible por hipoxia (HIF) en diversas células a través del cuerpo ${ }^{(7)}$.

La sobreexpresión del factor inducible por hipoxia (HIF) muestra un llamativo y progresivo incremento en el volumen óseo. Estas observaciones sugieren que el aumento de la expresión de HIF en osteoblastos expresa una mayor cantidad de factores angiogénicos que promueven la formación de hueso ${ }^{(10)}$.

Se ha reportado diversas respuestas de la hipoxia sobre cultivos celulares; estos determinan el incremento en la estabilización del HIF y la expresión de sus genes objetivos, entre los que destaca el factor de crecimiento vascular del endotelio (VEGF) ${ }^{(20,21,28,29)}$.

La expresión de uno de los genes maestros en la formación ósea -el RUNX2, que regula la síntesis de fosfatasa alcalina, sialoproteína ósea, colágeno tipo 1 , osteopontina y osteocalcina-, fue evaluada en cultivos celulares expuestos a un medio de hipoxia (1\% de $\left.\mathrm{O}_{2}\right)$ y a un medio de normoxia $(21 \%$ de $\mathrm{O}_{2}$ ). Se halló menor expresión del RUNX2 al evaluar a los 3 días el medio hipóxico comparado con la normoxia. También se determinó que la hipoxia tiene efecto inhibitorio sobre la mineralización en controles hechos a los 14 y 21 días ${ }^{(5)}$. Además, la disminución en la expresión de RUNX2, al exponer los cultivos celulares a $2 \%$ de $\mathrm{O}_{2}$ redujo la fosfatasa alcalina, el colágeno tipo 1 y la osteocalcina en todos los controles (3, 7, 14 y 21 días) comparados con normoxia $\left(21 \% \text { de } \mathrm{O}_{2}\right)^{(30)}$.
En la presente investigación, la menor formación ósea encontrada en el grupo mar 15 días comparado con el grupo altura 15 días, se debe a varios motivos. Entre estos destaca que la hipoxia tisular, en el grupo altura 15 días, logró una mayor estabilización de la subunidad citosólica HIF- $\alpha$ y su heterodimerización a nivel nuclear para formar el HIF, lo cual determina la expresión de múltiples genes; probablemente el más importante es el que codifica al factor de crecimiento vascular del endotelial (VEGF), debido a su implicancia en el proceso de osteogénesis, directamente activando a las células osteoblásticas o indirectamente mediante su potente acción angiogénica. Los antecedentes encontrados sugieren que la hipoxia inhibe al gen RUNX2 que, como se ha señalado, es un transcriptor de moléculas necesarias para la diferenciación y la función osteoblástica; aunque, por los resultados de la presente investigación, se entiende que la enérgica activación molecular de la vía del HIF fue la que determinó lo observado a los 15 días. Por otra parte, al activarse la angiogénesis y empezar las etapas de reparación ósea, la concentración de oxígeno en la zona injuriada, en el transcurso de los días, fue incrementándose, con lo cual la estabilización de la subunidad HIF- $\alpha$ fue disminuyendo (feedback negativo), expresando (en base al incremento de oxígeno tisular y a la menor expresión del HIF) vías moleculares que intervinieron en similares magnitudes en el proceso de formación ósea; esto puede explicar que a los 30 días ya no hubieran diferencias significativas. Finalmente, a medida que transcurrían los días en la zona injuriada, la cantidad de osteocitos fue cada vez mayor (mayor formación ósea); es entendible que el proceso de reparación se desarrolla en el tiempo, y a medida que transcurre este los mecanismos fisiológicos llevan hacia la madurez al tejido injuriado.

Se concluye que la presión de oxígeno ambiental influyó en la formación ósea, mejorando la proliferación de osteocitos en el grupo altura 15 días, al comparar el grupo mar 15 días versus el grupo altura 15 días. La presión de oxígeno ambiental no fue determinante significativo en la proliferación de osteocitos, al comparar el grupo mar 30 días versus el grupo altura 30 días. La formación ósea fue mayor a medida que transcurría más tiempo (mayor proliferación de osteocitos), en los dos ambientes evaluados.

\section{AGRADECIMIENTOS}

A las siguientes personas: Dr. Victor Chumpitaz Cerrate, Dra. Elydia Mujica Albán, Mg. Ronald Jiménez Aliaga, Mg. Martha Rodríguez Vargas, CD. Arturo Ramón Rosales.

\section{REFERENCIAS BIBLIOGRÁFICAS}

1. Gartner L, Hiatt J. Histologia Básica. 1era ed. Barcelona: Elsevier; 2011:84-9.

2. Gómez de Ferraris M, Campos Mu-oz A. Embriologia, Histologia e Ingenieria Tisular Bucodental. 3era ed. México DF: Editorial Médica Panamericana; 2009:381-7.

3. Wu E, Li H, Zhao T, Fan J, Ma X, Xiong L, et al. Effect of hypoxia on the gene profile of human bone marrow-derived mesenchymal stem cells. Sheng Li XueBao. 2007;59(2):227-32.

4. Villarruel S, Boehm C, Pennington M, Bryan J, Powel K, Muschlen G. The effect of oxygen tension on the in vitro assay of human osteoblastic connective tissue progenitor cells. J Orthop Res. 2008;26(10):1390-7. doi: http://dx.doi.org/10.1002/ jor.20666

5. Yang D, Yang M, Tsai C, Huang T, Chen Y, Hung $S$, et al. Hypoxia inhibits osteogenesis in human mesenchymal stem cells through direct regulation of RUNX2 by TWIST. PLoS One. 2011;6(9):e23965. doi: http://dx.doi.org/10.1371/ journal.pone.0023965

6. Mamalis AA, Cochran DL. The therapeutic potential of oxygen tension manipulation via hypoxia inducible factors and mimicking agents in guided bone regeneration. Arch Oral Biol. 2011;56(12):1466-75. doi: http://dx.doi. org/10.1016/j.archoralbio.2011.05.001

7. Semenza GL. Regulation of oxygen homeostasis by hypoxia-inducible factor 1 . Physiology. 2009;24:97-106. doi: http://dx.doi.org/10.1152/ physiol.00045.2008

8. Tang W, Yang F, Li Y, de Crombrugghe B, Jiao H, Xiao $\mathrm{G}$, et al. transcriptional regulation of vascular endothelial growth factor (VEGF) by osteoblastspecific transcription factor osterix (OsX) in osteoblasts. J Biol Chem. 2012;287(3):1671-8. doi: http:// dx.doi.org/10.1074/jbc.M111.288472

9. Aguirre E. Influencia de la hipoxia sobre el metabolismo óseo. Rol central del factor inducible por hipoxia. An Fac med. 2013;74(4):321-5.

10. Wan C, Shao J, Gilbert SR, Riddle RC, Long F, Johnson RS, et al. Role of HIF-1 alpha in skeletal development. Ann N Y Acad Sci. 2010;1192:322-6. 
doi: http://dx.doi.org/10.1111/j.1749-6632 .2009.05238.x

11. Bracken C, Fedele A, Linke S, Balrak W, Lissy $\mathrm{K}$, Whitelaw $\mathrm{M}$, et al. Cell-specific regulation of hypoxia-inducible factor (HIF)- $1 \alpha$ and HIF- $2 \alpha$ stabilization and transactivation in a graded oxygen environment. J Biol Chem. 2006;281(32):22575-85. doi: http://dx.doi.org/10.1074/jbc.M600288200

12. Wang Y, Wan C, Deng L, Liu X, Cao X, Gilbert S, et al. The hypoxia-inducible factor a pathway couples angiogenesis to osteogenesis during skeleta development. J Clin Invest. 2007;117(6):1616-26. doi: http://dx.doi.org/10.1172/JCl31581

13. Tsai Ch, Yew T, Yang D, Huang W, Hung S. Benefits of hypoxic culture on bone marrow multipotent stromal cells. Am J Blood Res. 2012;2(3):148-59.

14. Mohyeldin A, Garzón T, Qui-ones A. Oxygen in stem cell biology: a critical component of the stem cell niche. Cell Stem Cell. 2010;7(2):150-61. doi: http://dx.doi.org/10.1016/j.stem.2010.07.007

15. Holzwarth C, Vaegler M, Gieseke F, Pfister S, Handgretinger R, Kerst G, et al. Low physiologic oxygen tensions reduce proliferation and differentiation of human multipotent mesenchymal stromal cells. BMC Cell Biol. 2010;11:11. doi: http://dx.doi. org/10.1186/1471-2121-11-11

16. Utting J, Robins S, Brandao-Burch A, Orriss I, Behar J, Arnett T. Hypoxia inhibits the growth, differentiation and bone-forming capacity of rat osteoblasts. Exp Cell Res. 2006;312(10):1693-702. doi: http://dx.doi.org/10.1016/j.yexcr.2006.02.007

17. Wang Y, Li J, Wang Y, Lei L, Jiang Ch, An S, et al. Effects of hypoxia on osteogenic differentiation of rat bone marrow mesenchymal stem cells, Mol Cell Biochem. 2012;362(1-2):25-33. doi: http://dx.doi. org/10.1007/s11010-011-1124-7

18. Nicolaije C, Koedam M, van Leeuwen J. Decreased oxygen tension lowers reactive oxygen species and apoptosis and inhibits osteoblast matrix mi- neralization through changes in early osteoblast differentiation. J Cell Physiol. 2012;227(4):1309-18. doi: http://dx.doi.org/10.1002/jcp.22841

19. Grayson WL, Zhao F, Izadpanah R, Bunnell B, Ma $T$. Effects of hypoxia on human mesenchymal stem cell expansion and plasticity in 3D constructs. $J$ Cell Physiol. 2006;207(2):331-9. doi: http://dx.doi. org/10.1002/jcp.20571

20. Wagegg M, Gaber T, Lohanatha F, Hahne M, Strehl C, Fangradt M, et al. Hypoxia promotes osteogenesis but suppresses adipogenesis of human mesenchymal stromal cells in a hypoxiainducible factor-1 dependent manner. PLoSOne. 2012;7(9):e46483.

21. Razban V, Sahebqadam A, Khoshdel A. HIF$1 \alpha$ overexpression induce angiogenesis in mesenchymal stem cells. Biores Open Access. 2012;1(4):174-83. doi: http://dx.doi.org/10.1089/ biores.2012.9905

22. Cáceres L. Alteraciones en el crecimiento y desarrollo óseo en ratas albinas sometidas a dieta deficiente en proteinas y diferentes concentraciones de hierro [Tesis de Maestria]. Lima: Universidad Nacional Mayor de San Marcos; 2004

23. Lu Ch, Saless N, Wang X, Sinha A, Decker S, Kazakia $\mathrm{G}$, et al. The role of oxygen during fracture healing. Bone. 2013;52(1):220-9. doi: http://dx.doi. org/10.1016/j.bone.2012.09.037

24. Baldo H, Lima A, Monti I, Pires A. Estudio histológico comparativo de la reparación ósea entre hueso alveolar en los cerdos sometidos a osteotomia con alta y baja velocidad, con refrigeración líquida. Rev Esp Cir Oral Maxilofac. 2012;34(1):18-24.

25. Guide for the Care and Use of Laboratory Animals. Eight ed. Washington D.C.: National Academy Press; 2010.

26. Gad SC. Animal Models in Toxicology. 2doed. New York: Taylor \& Francis Group; 2007:354.
27. Fernandes M, Pinheiro P Cantarelli A, Marotta M. Densitometric analysis of the autogenous de mineralized dentin matrix on the dental socket wound healing process in humans [Análise densitométrica da matriz dentinária desmineralizada autógena na reparação alveolar de humanos]. Braz Oral Res. 2006;20(4):324-30.

28. Ishizuka T, Hinata T, Watanabe Y. Superoxide induced by a high-glucose concentration attenuates production of angiogenic growth factors in hypoxic mouse mesenchymal stem cells. J Endocrinol. 2011;208(2):147-59. doi: http://dx.doi.org/10.1677/ JOE-10-0305

29. Genetos D, Wong A, Watari S, Yellowley CE. Hypoxia increases annexin A2 expression in osteoblastic cells via VEGF and ERK. Bone. 2010;47(6):1013-9. doi: http://dx.doi.org/10.1016/j. bone.2010.08.024

30. Huang YC, Zhu HM, Cai JQ, Huang YZ, Xu J, Zhou $Y$, et al. Hypoxia inhibits the spontaneous calcification of bone marrow-derived mesenchymal stem cells. J Cell Biochem. 2012;113(4):1407-15. doi: http://dx.doi.org/10.1002/jcb.24014

Artículo recibido el 24 de octubre de 2013 y aceptado para publicación el 9 de febrero de 2014.

Financiamiento: Recursos propios del autor

No existen conflictos de intereses

Correspondencia:

Mg. Elias Ernesto Aguirre Siancas

Dirección: Jr. Pomacanchi 326. Lima 30, Perú

Teléfonos: 323-3612 y 9979-38372

Correo: elias_aguirre@yahoo.com 